\title{
Elevated nitrate enriches microbial functional genes for potential bioremediation of complexly contaminated sediments
}

\author{
Meiying Xu, Qin Zhang, Chunyu Xia, Yuming Zhong, Guoping Sun, Jun Guo, Tong Yuan, \\ Jizhong Zhou and Zhili He
}

The ISME Journal (2015) 9, 532; doi:10.1038/ismej.2014.229

Correction to: The ISME Journal (2014) 8, 1932-1944; doi:10.1038/ismej.2014.42; published online 27 March 2014

Since the publication of this article, the authors have identified an error in the Acknowledgements section. The correct acknowledgements are as follows:

\section{Acknowledgements}

We thank Dr Daiyong Deng for field work and Dr James W Voordeckers for improving the English language. This research was supported by the
National Basic Research Program of China (973 Program) (2012CB22307), the National Natural Science Foundation of China (31170470), Guangdong Province-Chinese Academy of Sciences Strategic Cooperative Project (2012B091100257), the International Cooperation Projects of Guangdong Province (2011B050400005), Guangdong Provincial Programs for Science and Technology Development (2012A061100009) and Guangdong Provincial Innovative Development of Marine Economy Regional Demonstration Projects (GD2012-D01-002).

The authors would like to apologise for any inconvenience this may have caused.

\section{Viruses accumulate in aging infection centers of a fungal forest pathogen}

Eeva J Vainio, Michael M Müller, Kari Korhonen, Tuula Piri and Jarkko Hantula

The ISME Journal (2015) 9, 532; doi:10.1038/ismej.2014.181

Correction to: The ISME Journal (2015) 9, 497-507; doi:10.1038/ismej.2014.145; published online 15 August 2014

Since the publication of this article, the authors have identified an error in the acknowledgements section of their paper, namely that the following decision numbers were omitted from the funding organization: Academy of Finland (decision numbers 251193 and 258520).

The corrected acknowledgements are as follows:

\section{Acknowledgements}

This study was financially supported by the Academy of Finland (decision numbers 251193 and 258520) and the Finnish Forest Research Institute. We thank Marja-Leena Santanen, Juha Puranen, Nieves Lorenzo Gotor, Heidi Ylinenpää, Sonja Sarsila and Suvi Saloranta for technical assistance and Jaana Jurvansuu and Taina Pennanen for commenting on the manuscript.

This has now been added to the acknowledgements, and the corrected article appears in this issue. The html and online pdf versions have also been rectified, and now carry the correct paper.

The authors would like to apologize for any inconvenience this may have caused. 